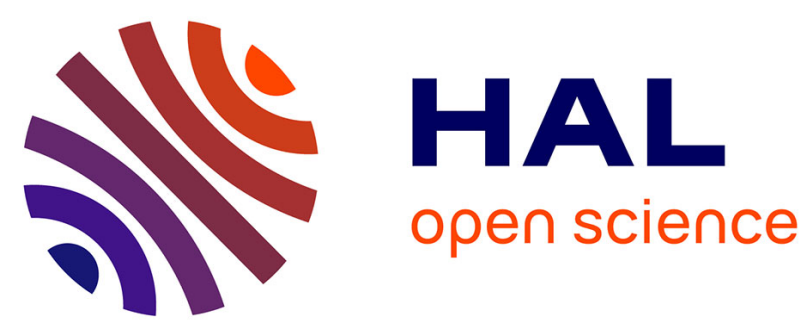

\title{
The arabidopsis chloroplast ribosomal protein L21 is encoded by a nuclear gene of mitochondrial origin
}

\author{
Jean-Luc Gallois, P. Achard, G. Green, R. Mache
}

\section{To cite this version:}

Jean-Luc Gallois, P. Achard, G. Green, R. Mache. The arabidopsis chloroplast ribosomal protein L21 is encoded by a nuclear gene of mitochondrial origin. Gene, 2001, 274 (1-2), pp.179-185. 10.1016/S03781119(01)00613-8 . hal-01594066

\section{HAL Id: hal-01594066 https://hal.science/hal-01594066}

Submitted on 26 Sep 2017

HAL is a multi-disciplinary open access archive for the deposit and dissemination of scientific research documents, whether they are published or not. The documents may come from teaching and research institutions in France or abroad, or from public or private research centers.
L'archive ouverte pluridisciplinaire HAL, est destinée au dépôt et à la diffusion de documents scientifiques de niveau recherche, publiés ou non, émanant des établissements d'enseignement et de recherche français ou étrangers, des laboratoires publics ou privés. 


\title{
The Arabidopsis chloroplast ribosomal protein L21 is encoded by a nuclear gene of mitochondrial origin ${ }^{\text {is }}$
}

\author{
Jean-Luc Gallois ${ }^{1}$, Patrick Achard, Gaynor Green, Régis Mache* \\ Laboratoire de Génétique Moléculaire des Plantes, Université J. Fourier and Centre National de la Recherche Scientifique, \\ BP 53, F-38041 Grenoble Cedex 09, France
}

Received 20 December 2000; received in revised form 6 June 2001; accepted 11 July 2001

Received by W. Martin

\begin{abstract}
Many chloroplast genes of cyanobacterial origin have been transferred to the nucleus during evolution and their products are re-addressed to chloroplasts. The RPL21 gene encoding the plastid r-protein L21 has been lost in higher plant chloroplast genomes after the divergence from bryophytes. Based on phylogenetic analysis and intron conservation, we now provide evidence that in Arabidopsis a nuclear RPL21c gene of mitochondrial origin has replaced the chloroplast gene. The control of expression of this gene has been adapted to the needs of chloroplast development by the acquisition of plastid-specific regulatory promoter cis-elements. (C) 2001 Elsevier Science B.V. All rights reserved.
\end{abstract}

Keywords: RPL21 genes; Higher plants; Phylogeny; Gene expression; Promoter cis-elements

\section{Introduction}

Molecular data and phylogenetic results concur to support the hypothesis of a cyanobacterial origin of chloroplasts. After the engulfment of a cyanobacterium in a primitive eukaryotic cell cyanobacterial genes were either lost or transferred into the host genome, resulting in the structure of the present chloroplast chromosome. The organization of this chromosome is remarkably conserved in all evolutionary distant lineages, from algae to angiosperms, giving evidence that the transfer of cyanobacterial genes to the nucleus occurred rapidly after endosymbiosis. However, a few genes were transferred much later, including components of the translational apparatus. For instance, the tufA gene, encoding plastid elongation factor $\mathrm{Tu}$, is present in chloroplasts of algae and has been transferred to the nucleus in an algal lineage that gave rise to land plants (Baldauf and Palmer, 1990). The rpl22 gene coding for the

\footnotetext{
Abbreviations: r-protein, ribosomal protein; RT-PCR, reverse transcriptase-polymerase chain reaction

The nucleotide sequence data reported here appeared in the EMBL databank under the Accession numbers Y15964 and AJ278909.

* Corresponding author. Tel.: +33-4-76-51-46-67; fax: +33-4-76-51-4336.

E-mail address: regis.mache@ujf-grenoble.fr (R. Mache).

${ }^{1}$ Present address: Department of Cellular and Developmental Biology, John Innes Centre, Norwich, NR4 7UH, UK.
}

ribosomal protein (r-protein) L22 represents another interesting example of late transfer. In legumes, the rpl22 gene has been transferred into the nucleus, possibly 100 million years before the loss from the chloroplast genome (Gantt et al., 1991). During this time the nuclear $r p l 22$ gene has been preserved suggesting that it was active. In a recent study, Martin et al. (1998) identified 44 functional nuclear genes out of 210 genes examined that originated from a cyanobacterial ancestral genome and whose products are addressed to chloroplasts. This means that present-day chloroplast proteins are mainly of cyanobacterial origin, regardless of where the corresponding genes are localized, in plastids or in the nucleus. Because the mitochondrial genome originated from an endosymbiotic $\alpha$-proteobacterium (Andersson et al., 1998; Gray et al., 1999), the same principle should be true for mitochondrial proteins. Taken together, the question arises whether present-day nuclear genes of cyanobacterial origin could also provide proteins that are relocated to mitochondria and/or whether nuclear genes of $\alpha$-proteobacterial origin could provide proteins that are relocated to plastids. In the present paper we addressed the second part of this question. The RPL21 gene is still present in the chloroplast chromosome of many algae and the lower land plant Marchantia polymorpha, a bryophyte, but is lost from the chloroplast genomes of higher plants. Based on the relative low conservation of chloroplast L21 protein sequences between Marchantia and spinach, it was proposed several 
years ago that the spinach nuclear gene might be of mitochondrial origin (Martin et al., 1990). But phylogenetic analyses were lacking to support the hypothesis. Detailed analysis of prokaryotic-type RPL21 genes in Arabidopsis thaliana, a higher plant, shows that the nuclear gene replacing the chloroplast gene is not related to cyanobacterial species and is grouped with the Caenorhabditis elegans mitochondrial L21-like gene. The expression of this gene has been adapted to chloroplast needs during evolution as demonstrated by the existence of chloroplast r-proteinspecific regulatory elements in the promoter of this gene.

\section{Materials and methods}

\subsection{Cloning and sequence analyses}

An Arabidopsis genomic library (ecotype Columbia) has been screened with the $\alpha-{ }^{32} \mathrm{P}$-labeled EST 146E8T7 (Accession number: T75800) that covers two-thirds of the RPL21c cDNA. Standard procedures (Sambrook et al., 1989) were used for genomic DNA isolation, subcloning into pBluescript II (Stratagene), RNA isolation, hybridization and sequencing, using an Applied Biosystems 373 XL automated DNA sequencer.

\section{2. $R T-P C R$}

Arabidopsis RNA (1-3 $\mu \mathrm{g})$ was used for RT-PCR. Primers were as follows: adenosine phosphoribosyl transferase, APT, CCTTTCCCTTAAGCTCTG, TCCCAGAATCGCTAAGATTGCC; $R P L 21 \mathrm{c}$, AGAGGATCCCAAAGTCTCGAGCTTAGAG, GAATGGCAAAAGGTACA; $R P$ L21m, TCGTGCTCTCCGATGGCG, AGGCCTTTTCATCATTTC. The $1 \mathrm{~kb}$ ladder (Gibco-BRL) was used as a size marker.

\subsection{Gel shift assays}

Gel retardation analyses were performed as described by Lagrange et al. (1997) using 30-60 $\mu \mathrm{g}$ Arabidopsis whole protein extracts and the following annealed oligonucleotides specific to elements of the RPL21c promoter. Monomers of the S2 site, dimers of GT1 and S1 sites or mutants (GT1M and S1M) were used: S2, 5' -TAAGTAATTTTGTTATAATACAAACTGTGA- $3^{\prime}$ and $3^{\prime}$-TTAAAACAATATTATGTTTGACACTTTGACA-5 ${ }^{\prime}$; GT1 1 , 5'-AAACCAATTTGAA-3' and $3^{\prime}$-TTTGGTTAAACTT-5'; GT1M, 5'-AAAGGAATTTGAA- $3^{\prime}$ and $3^{\prime}$-TTTCCTTAAACTT- $5^{\prime}$; S1, 5'GGGGTACAATGGTAATTTT- $3^{\prime}$ and $3^{\prime}$-CCCCATGTTACCATTAAAA- $5^{\prime}$; and S1M, 5'-GGGGTAGAGCTGTAATTTT- $3^{\prime}$ and $3^{\prime}$-CCCCATCTCGACATTAAAA- $5^{\prime}$. The fragments were $\left[\alpha-{ }^{32} \mathrm{P}\right] \mathrm{CTP}$-labeled and gel-purified. Electrophoresis was performed on $6 \%$ non-denaturing polyacrylamide gels.

\subsection{Computer analyses}

Sequences were analyzed using the GCG software (University of Wisconsin) and internet-available bioinformatic programs. Parsimony, neighbor-joining and FitchMargoliash tree building were performed through the Pasteur (http://bioweb.pasteur.fr) and Infobiogen (http://www.infobiogen.fr) servers. Trees were drawn with the Phylodendron tree printer program at http://iubio.bio.indiana.edu/. The L21 r-protein sequences from prokaryotes and the prokaryoticlike L21 r-protein sequences from plants were retrieved from available databanks. The different sequences were aligned using the GCG pileup program and the conserved core sequences were used for phylogenetic analysis. Because the L21 protein deduced from a Physcomitrella patens EST (Accession number: AW145090) is too short it was not included in the data used for phylogenetic tree building. The sequences corresponding to the cytosolic $60 \mathrm{~S}$ r-protein L21 are not included because they present no similarities with L21 r-proteins from either chloroplast or from bacteria. Prediction of organelle localization was made using the following web sites: http://www.cbs.dtu.dk/services/ targetP/; http://www.cbs.dtu.dk/services/chloroP/; http:// www.psort.nibb.ac.jp:8800/.

\section{Results}

3.1. The Arabidopsis genome contains two RPL21 genes of prokaryotic origin

An Arabidopsis genomic clone was isolated using Arabidopsis EST encoding the 50S chloroplast r-protein L21 as a probe. After sequencing, the corresponding gene, termed $R P L 21 \mathrm{c}$, and the adjacent genes were identified (Accession number: Y15964). The adjacent genes code for a Ca-dependent protein kinase (Accession number: D21806) and a phosphoenolpyruvate carboxykinase (Accession number: CAB38935). The RPL21c gene is located on chromosome 1 (Accession number: gi6289106). This gene is unique in Arabidopsis as verified by Southern blot hybridization (data not shown) and it is functional as shown by the isolation of the corresponding cDNA clone (Accession number: Z49787). It is orthologous to the spinach chloroplast RPL21 gene (76\% identity and $86 \%$ similarity). A second putative gene located on chromosome 4 was sequenced and annotated in the framework of the Arabidopsis Genome Initiative (Accession number: gi7269995). This gene encodes SP8-like protein that includes a L21 r-protein signature. We re-examined the sequence data and found that the putative gene can be separated into two different genes, one encoding a L21 r-protein, the other one encoding the SP8-like protein. We termed the L21-encoding gene RPL21m (Accession number: AJ278909) to distinguish it from RPL21c. One EST (Accession number: AI995545) corresponding exclusively to exons of the $R P L 21 \mathrm{~m}$ gene 


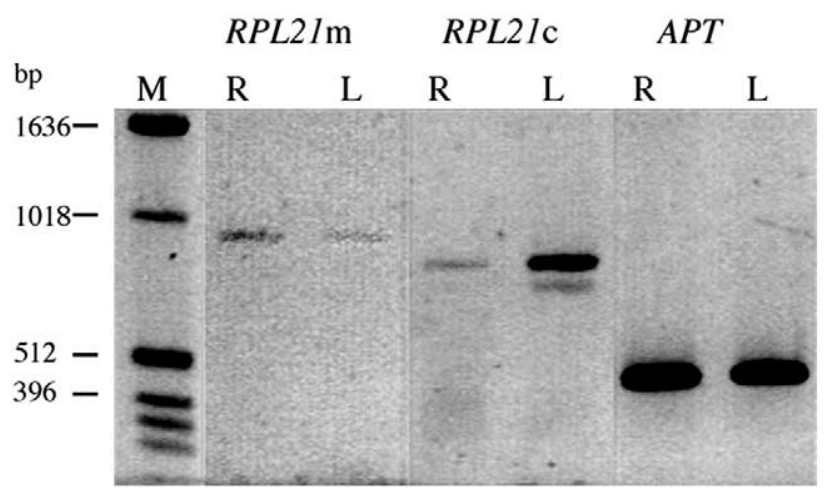

Fig. 1. Expression of RPL21 genes in Arabidopsis. Semi-quantitative measurement of mRNAs is made by RT-PCR (27 cycles). Primers corresponding to RPL21c, RPL21m and constitutively expressed adenosine phosphoribosyl transferase gene, $A P T$, taken as a control, were used. cDNAs were analyzed by electrophoresis using RNAs isolated from roots (R) or leaves (L). M, size markers.

is present in the dbEST databank, supporting our hypothesis that the RPL21m gene is expressed independently of the SP8-like gene. In order to confirm the existence of RPL21m mRNA, we performed Northern and RT-PCR analyses. Northern analysis revealed that RPL21m encodes a transcript of the expected size $(0.9 \mathrm{~kb}$; data not shown).
The RT-PCR analyses show that both the RPL21m and the $R P L 21 \mathrm{c}$ genes are constitutively expressed, but that only the $R P L 21 \mathrm{c}$ gene is highly expressed in leaves (Fig. 1). From these data, we conclude that two independent $R P L 21$ genes exist in A. thaliana.

Computer analysis of the Arabidopsis RPL21 genes using different algorithms (see Section 2) clearly predicts a chloroplast-targeting N-terminal sequence for the RPL21c gene product and a mitochondrial-targeting $\mathrm{N}$-terminal sequence for the RPL21m gene product. It was previously shown by Sanchez et al. (1996) that antibodies directed against the orthologous spinach chloroplast L21 protein recognized an Arabidopsis chloroplast protein of the same size. These antibodies do not cross-react with mitochondrial proteins, thus confirming the chloroplast localization of the RPL21c gene product. We assume that $R P L 21 \mathrm{~m}$ encodes a mitochondrial protein because only one $\mathrm{L} 21$ protein exists per organelle and $\mathrm{L} 21 \mathrm{~m}$ protein is closer than L21c protein to the mitochondrial protein of C. elegans (32\% identity versus $28 \%$ ).

\subsection{The chloroplast RPL21c gene is of mitochondrial origin}

The comparison of the RPL21c and RPL21m proteins with the spinach plastid L21 r-protein and the L21 proteins

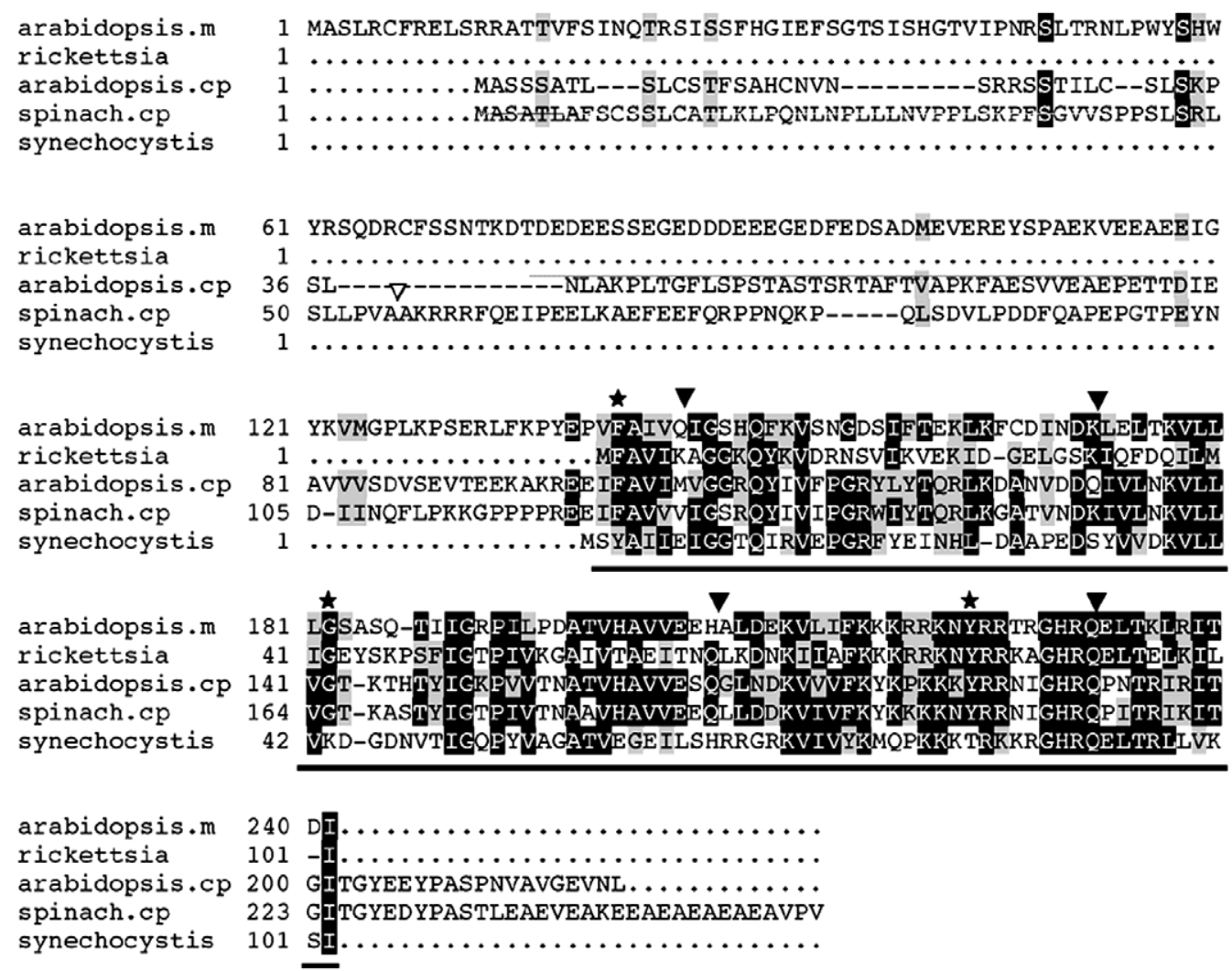

Fig. 2. Amino acid sequence alignment of chloroplast or mitochondrial L21 r-proteins from spinach and Arabidopsis with their respective eubacterial ancestors, Synechocystis sp. and Rickettsia prowazekii, respectively. Spinach-cp, Spinacia oleracea chloroplast; Arabidopsis-cp, A. thaliana chloroplast; Arabidopsis-m, A. thaliana mitochondria. Corresponding positions of introns in the nucleotide sequence of higher plant RPL21 genes are indicated (black triangles). The cleavage site leaving the mature L21 in spinach chloroplast is reported (open triangle). The conserved prokaryotic core of L21 proteins is underlined. The two amino acids specific to the $\alpha$-proteobacterial lineage are indicated by a star. Identical amino acids are boxed in black, and similar amino acids are boxed in gray. 
from ancestors of chloroplast (a cyanobacterium, Synechocystis sp.) or of mitochondria (an $\alpha$-proteobacterium, Rickettsia prowazekii) is shown in Fig. 2. The higher plant L21 rproteins have conserved a central core similar to the bacterial L21 amino acid sequences. In the N-terminal region, the plant L21 proteins have acquired a targeting sequence followed by a long protein extension not significantly related to each other, probably because the selection pressure on sequences encoding these elements was not very high. In contrast to higher plants, the L21 r-protein of the lower plant $M$. polymorpha is encoded in the chloroplast genome and is much more similar to the cyanobacterial L21 protein, lacking the $\mathrm{N}$-terminal extension and resulting in a similar size (data not shown).

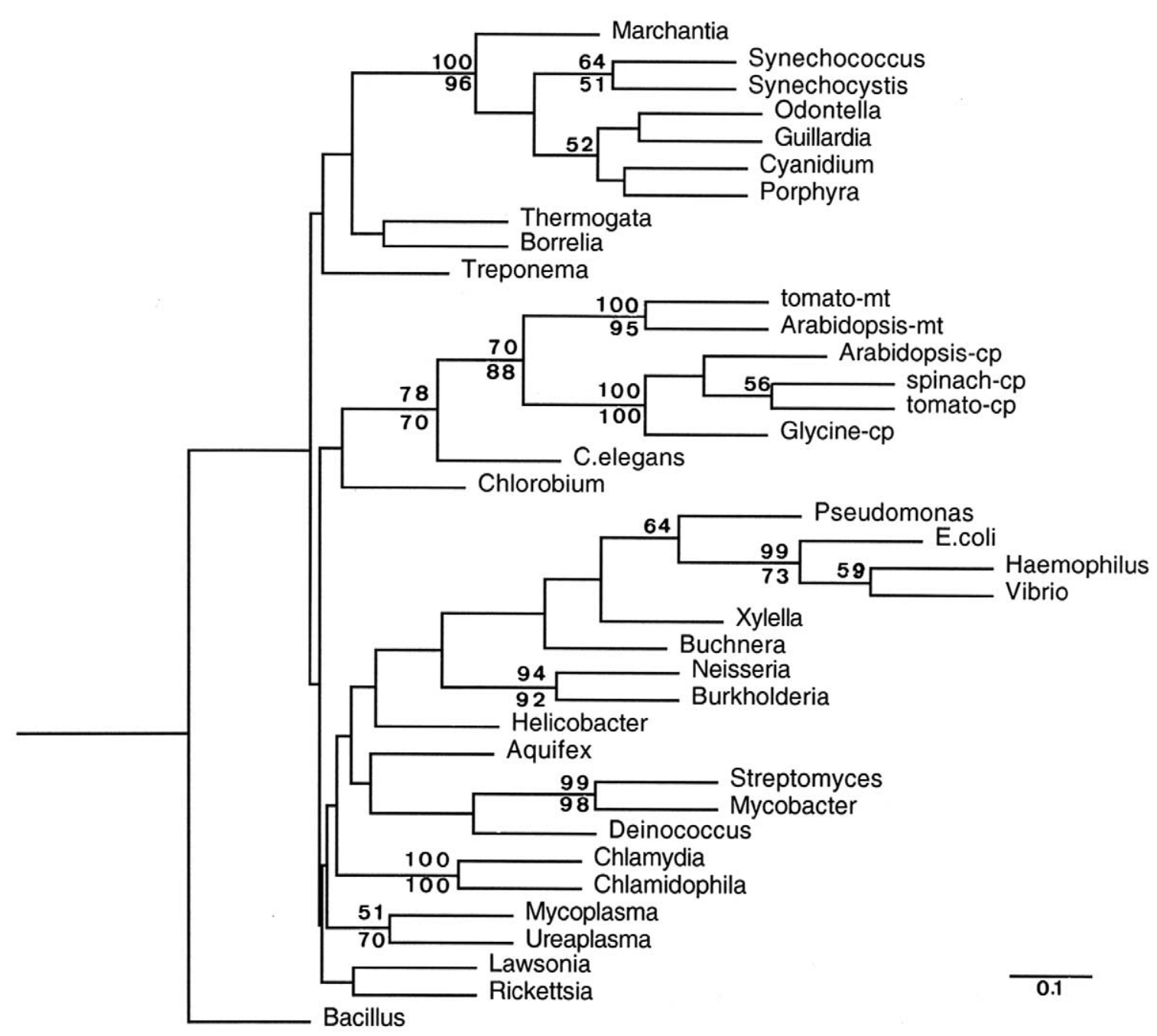

Fig. 3. Protein phylogeny of chloroplast and mitochondrial L21 r-proteins. The unrooted tree was constructed by the Fitch-Margoliash least-square distance method using 50S L21 r-proteins from plants, from bacteria and from C. elegans. The core regions of amino acid sequences (Fig. 2) have been used. The L21 protein sequences and their Accession numbers are as follows. (1) Lower plants, chloroplast-encoded: Cyanidium caldarium, Cyanidium, a rhodophyte, AF022186; Porphyra purpurea, Porphyra, a rhodophyte, P51209; Guillardia theta, Guillardia, a cryptophyte, O78429; Marchantia polymorpha, Marchantia, a bryophyte, P06387. (2) Higher plants, nuclear-encoded: Spinacia oleracea, Spinacia-cp, P24613; Arabidopsis thaliana, Arabidopsis-cp, P51412; Arabidopsismt, deduced from AL161578; Lycopersicum esculentum, Tomato-cp, EST AW031100, and Tomato-mt, EST AI485205; Glycine maxima, Glycine-cp, AW831287; Glycine-mt, EST AW234792. (3) Animals: Caenorhabditis elegans, C. elegans, Z46937. (4) Bacteria: Aquifex aeolicus, Aquifex, AAC07544; Bacillus subtilis, Bacillus, CAA42108; Borrelia burgdorferi, Borrelia, AAV67129; Buchnera sp., Buchnera, BAB13091; Burkholderia pseudomallei, Burkholderia, AAG01347; Chlamydia trachomatis, Chlamydia, AAC68017; Chlamydophila pneumoniae, Chlamidophila, AAF38077; Chlorobium vibrioforme, Chlorobium, CAA55804; Deinococcus radiodurans, Deinococcus, AAF09679; Escherichia coli, E. coli, AE000399; Haemophilus influenzae, Haemophilus, AAC22536; Helicobacter pylori, Helicobacter, AAD07365; Lawsonia intracellularis, Lawsonia, AAC67129; Mycobacterium tuberculosis, Mycobacter, CAB03777; Mycoplasma pneumoniae, Mycoplasma, AAB961591; Neisseria meningitidis, Neisseria, AL162758; Pseudomonas aeruginosa, Pseudomonas, AAG01347; Rickettsia prowazekii, Rickettsia, CAA15179; Streptomyces coelicolor, Streptomyces, CAB75378; Synechococcus sp., Synechococcus, BAA3700; Synechocystis sp., Synechocystis, P74266; Thermogata maritima, Thermogata, AAD36526; Treponema pallidium, Treponema, AAC65713; Ureaplasma urealyticum, Ureaplasma, AAF306201; Vibrio cholerae, Vibrio, AAF93608; Xylella fastidiosa, Xylella, AAF85223. Bootstrap values above 50, using 100 replications, using Fitch-Margoliash (upper) or parsimony (under) methods are indicated. The scale bar indicates 0.1 amino acid substitutions per site. 
To examine the origin of chloroplast L21 protein in different plant species, we compared the amino acid sequences of the conserved core regions. RPL21c is closer to RPL $21 \mathrm{~m}$ ( $46 \%$ identities) than to the chloroplast-encoded L21 protein of $M$. polymorpha (31\%). Using parsimony, neighbor-joining and Fitch-Margoliash least-square distance methods, phylogenetic trees that include prokaryotic and prokaryotic-like L21 sequences were constructed using 103 positions common to 38 different species. Protein sequences of cytoplasmic $60 \mathrm{~S} \mathrm{L21} \mathrm{r-proteins} \mathrm{that} \mathrm{possess} \mathrm{a}$ different signature than prokaryotic L21 r-proteins were not included in the trees. Only the Fitch-Margoliash tree is shown (Fig. 3). Results indicate that plant RPL21 r-proteins belong to two different clades. Similar results were obtained using parsimony or neighbor-joining methods of analysis. High bootstrap values were obtained by using the different methods of phylogenetic analyses showing that the chloroplast RPL21 genes of lower plants (algae and the bryophyte $M$. polymorpha) have a different origin than the nuclear RPL21 genes encoding the chloroplast L21 r-protein in higher plants. The clade of lower plants is clearly of cyanobacterial origin as expected. The clade of higher plants includes mitochondrial L21 r-proteins suggesting a mitochondrial origin. Interestingly, the recently sequenced genome of the worm C. elegans encodes a L21-like protein (Accession number: CAA87060) that is included in the mitochondrial group and that shares a common origin with higher plant L21 r-proteins. These results strongly suggest a mitochondrial origin for the higher plant clade and consequently for genes encoding chloroplast L21. Independently of these phylogenetic aspects, we analyzed higher plant RPL21 gene organization. Genes encoding either mitochondrial or chloroplast L21 gene products possess common features. The five exons and four introns are present at exactly the same position in the RPL21m and $R P L 21 \mathrm{c}$ genes (Fig. 2), indicating a common origin. We also searched for specific residues, unique to the products of nuclear genes of mitochondrial origin. Analysis of the sequence data of the 38 species used for phylogenetic tree construction (Fig. 3) showed that the F, G and Y residues that are indicated in the sequences reported in Fig. 2 are constantly and specifically present in the products of RPL21 genes of mitochondrial origin. These three residues might represent the signature of these genes. Altogether, the results show a mitochondrial origin for higher plant nuclear genes encoding chloroplast RPL21 proteins.

\subsection{Promoter elements controlling the expression of the RPL21c gene}

The replacement of the chloroplast gene product by a product of a nuclear gene of mitochondrial origin raises the question of the control of gene expression in order to adapt the synthesis of the RPL21c gene to chloroplast development in mesophyll cells, in parallel with leaf development. As shown in Fig. 1, the mitochondrial RPL $21 \mathrm{~m}$ gene shows a different expression pattern compared to the chloroplast RPL21c gene. The RPL21c is preferentially expressed in photosynthetic tissues. Several cis-acting elements have been characterized in promoters of nuclear genes encoding plastid proteins. Among these elements are the GT1 box (for review see Zhou, 1999), and the S1 and S2 sites (Villain et al., 1994; Lagrange et al., 1997). Sequences corresponding to these three sites are also present in the promoter region of the RPL21c gene as indicated in Table 1. These elements bind trans-acting factors present in Arabidopsis as shown by gel shift assays using appropriate probes (Fig. 4). The protein/DNA interactions are specific as shown by probing with mutated S1 site and mutated GT1 box or by competition with cold $\mathrm{S} 2$ site probe. Altogether, these experiments show that the RPL21c gene has acquired regulatory elements found in other nuclear genes encoding chloroplast r-proteins.

\section{Discussion}

Results presented in this paper indicate a mitochondrial origin for the nuclear RPL21c gene encoding a chloroplast $\mathrm{r}$ protein in Arabidopsis. Phylogenetic results and intron positions support this conclusion. RPL21c is distant from the $M$. polymorpha chloroplast-encoded $r p l 21$ gene and bootstrap analysis places $R P L 21 \mathrm{c}$ outside the entire lineage of chloroplast-encoded genes and concomitantly from their cyanobacterial ancestor. In contrast, $R P L 21 \mathrm{c}$ is placed in a sister clade with a group of mitochondrial origin. Mitochondria descend from an eubacteria different from cyanobacteria and related to the $\alpha$-proteobacterial group. Rickettsia prowazekii represents one of the closest eubacterial

Table 1

Common promoter cis-elements of the spinach RPL21, RPS1, and of Arabidopsis RPL21c genes ${ }^{\mathrm{a}}$

\begin{tabular}{lcc}
\hline Origin & S1 site & S2 site \\
\hline Spinach RPS1 (1) & 227 -TTCATGGTAACA-248 & 254 -CACATACACCT-264 \\
Spinach RPL21 (2) & 163 -CAAATGGTATTA-152 & 302 -CCCATACATTA-292 \\
& 137 -CAAATGGTATTA-126 & \\
A. thaliana RPL21c (3) & 78 -ACAATGGTATT-68 & 429-ATAATACAAAC-419 \\
& & 243 -TAAATACACTA-233 \\
\hline
\end{tabular}

\footnotetext{
${ }^{a}$ Sequences are numbered from the transcription start site, using promoter P2 (Lagrange et al., 1997). (1) Villain et al. (1994); (2) Lagrange et al. (1997); (3) Accession number: gi6289106. The S1 and GT1 boxes in the spinach RPS1 promoter have similar binding properties (Villain et al., 1994).
} 


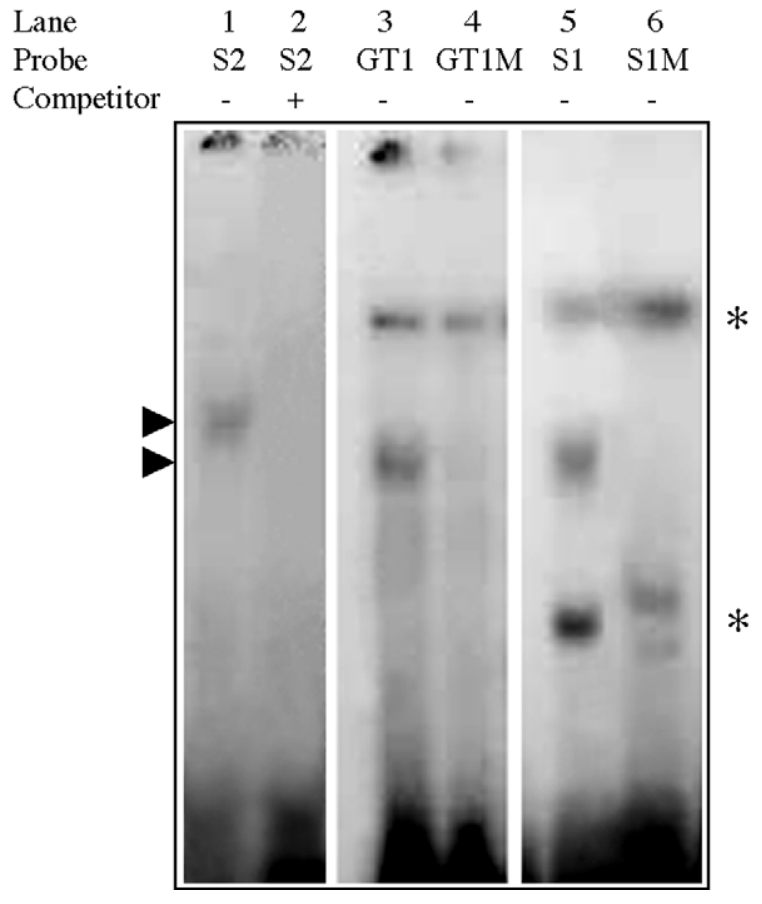

Fig. 4. Gel shift assays for Arabidopsis S2, GT1 and S1 sites. The labeled S2 site fragments (lanes 1 and 2), GT1 box and S1 site dimers (lanes 3 and 5) or mutated GT1M and S1M dimers (lanes 4 and 6) were incubated with protein extracts. S2 site fragments were incubated in the absence $(-)$ or presence $(+)$ of a 100-fold molar excess of unlabeled wild-type fragments (lanes 1 and 2). Protein/DNA complexes are indicated by arrowheads. The asterisks indicate non-specific bands.

genomes to mitochondria (Andersson et al., 1998). In our phylogenetic analyses, the L21 of $R$. prowazekii is not closely related to the higher plant L21 clade. This is probably related to the lack of accuracy in the branching of different bacterial groups as verified by the very low bootstrap values obtained after introducing 100 replicates in the different tree constructions. This is indeed related to the fact that bacterial phylogeny can not be determined by using a short protein as L21 only.

The RPL21c gene is present in one copy, as for other genes encoding plastid r-proteins. Its localization on chromosome 1 suggests that the RPL21c gene originated from a duplication of the $R P L 21 \mathrm{~m}$ gene located on chromosome 4 or from a polyploidization event as recently suggested (Arabidopsis Genome Initiative, 2000). The neighbor genes surrounding $R P L 21 \mathrm{~m}$ (a SP8-like gene and an unknown gene) have not been conserved during this duplication or polyploidization.

The replacement of a chloroplast-encoded gene product by a nuclear-encoded gene product requires a number of steps including the acquisition of a sequence for chloroplast targeting. In addition, in the case of RPL21c, the original mitochondrial exons had to be adapted to code for a protein that fits the chloroplast ribosome architecture.

We have considered the question of expression of a gene formerly adapted to mitochondria and whose product is now destined to chloroplasts. As shown in Fig. 1, $R P L 21 \mathrm{~m}$ is constitutively expressed in different tissues. The promoter regions of the RPL21c and RPL $21 \mathrm{~m}$ genes are not conserved (data not shown). To adapt the expression to chloroplasts the promoter region of the RPL21c gene must have acquired several specific cis-elements. Interestingly, these elements are present in several nuclear genes encoding chloroplast r-proteins of spinach and Arabidopsis (Zhou, 1999; Lagrange et al., 1997) suggesting that coordinated synthesis of different plastid r-proteins is made via similar cis-elements.

The recruitment of a nuclear gene of mitochondrial origin to replace the function of a formerly chloroplast-located gene is unusual. It probably represents an exception to the rule. The products of many genes of cyanobacterial origin that are encoded in the nucleus are re-located to plastids (Martin et al., 1998; Martin and Herrmann, 1998). The reason for the exceptional channeling of nuclear gene of mitochondrial origin to chloroplasts remains speculative. However, regarding the quantity of genes of cyanobacterial origin that have been successfully transferred to the nucleus during evolution, one can easily envisage that some transfer events were unsuccessful, i.e. the integration of the gene into the nuclear DNA did not occur in a region favorable for the linkage of a sequence encoding a plastid transit peptide necessary for the transport of the protein to the plastid. In such a case, replacement by a gene of mitochondrial origin becomes possible, if gene duplication, integration of the duplicated gene in a favorable context and compatibility of function are assured.

\section{Acknowledgements}

We thank Gérard Clabault for advice and help, and Professor N.-H. Chua for the gift of the GT1 box (BoxII) and mutated GT1M box oligonucleotides. We acknowledge the financial support of Région Rhône-Alpes (Programme "La Semence") and of the EEC (ESSA 3 Programme).

\section{References}

Andersson, S.G., Zomorodipour, A., Andersson, J.O., Sicheritz-Ponten, T., Alsmark, U.C., Podowski, R.M., Naslund, A.K., Eriksson, A.S., Winkler, H.H., Kurland, C.G., 1998. The genome sequence of Rickettsia prowazekii and the origin of mitochondria. Nature 396, 133-140.

Arabidopsis Genome Initiative, 2000. Analysis of the genome sequence of the flowering plant Arabidopsis thaliana. Nature 408, 796-815.

Baldauf, S.L., Palmer, J.D., 1990. Evolutionary transfer of the chloroplast tufA gene to the nucleus. Nature 344, 262-265.

Gantt, J.S., Baldauf, S.L., Calie, P.J., Weeden, N.F., Palmer, J.D., 1991. Transfer of $\mathrm{rpl} 22$ to the nucleus greatly preceded its loss from the chloroplast and involved the gain of an intron. EMBO J. 10, 3073-3078.

Gray, M.W., Burger, G., Lang, B.F., 1999. Mitochondrial evolution. Science 283, 1476-1481.

Lagrange, T., Gauvin, S., Yeo, H.J., Mache, R., 1997. S2F, a leaf-specific 
trans-acting factor, binds to a novel cis-acting element and differentially activates the RPL21 gene. Plant Cell 9, 1469-1479.

Martin, W., Herrmann, R.G., 1998. Gene transfer from organelles to the nucleus: how much, what happens, and why? Plant Physiol. 118, 917.

Martin, W., Stoebe, B., Goremykin, V., Hansmann, S., Hasegawa, M., Kowallik, K., 1998. Gene transfer to the nucleus and the evolution of chloroplasts. Nature 393, 162-165.

Martin, W., Lagrange, T., Li, Y.F., Bisanz-Seyer, C., Mache, R., 1990. Hypothesis for the evolutionary origin of the chloroplast ribosomal protein L21 of spinach. Curr. Genet. 18, 553-556.

Sambrook, J., Maniatis, T., Fritsch, E.F., 1989. Molecular Cloning: A
Laboratory Manual. Cold Spring Harbor Laboratory Press, Cold Spring Harbor, NY.

Sanchez, H., Fester, T., Kloska, S., Schroder, W., Schuster, W., 1996. Transfer of rps19 to the nucleus involves the gain of an RNP-binding motif which may functionally replace RPS13 in Arabidopsis mitochondria. EMBO J. 15, 2138-2149.

Villain, P., Clabault, G., Mache, R., Zhou, D.X., 1994. S1F binding site is related to but different from the light-responsive GT-1 binding site and differentially represses the spinach rps1 promoter in transgenic tobacco. J. Biol. Chem. 269, 16626-16630.

Zhou, D.X., 1999. Regulatory mechanism of plant gene transcription by GT-elements and GT-factors. Trends Plant Sci. 4, 210-214. 